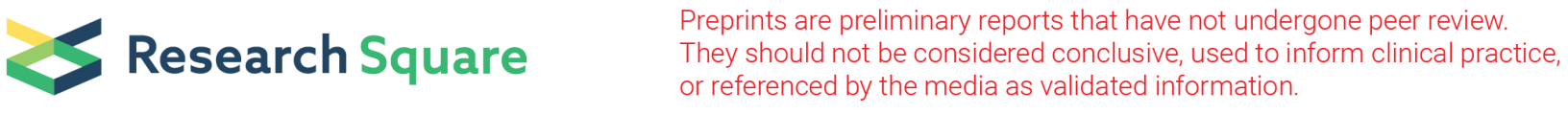

\title{
Role of Growth Factors \& Neurophysiological Markers In Evaluation of Neuroplasticity Using 1Hz rTMS With Physical Therapy In Ischemic Stroke: A Double Blind, Parallel Group, Sham Controlled Randomized Trial
}

\author{
Hina Sharma \\ All India Institute of Medical Sciences \\ Nand Kumar \\ All India Institute of Medical Sciences \\ Sreenivas Vishnubhatla \\ All India Institute of Medical Sciences \\ Rajeswari MR \\ All India Institute of Medical Sciences \\ M.V.Padma Srivastava ( $\square$ vasanthapadma123@gmail.com ) \\ All India Institute of Medical Sciences
}

Research Article

Keywords: stroke, neuroplasticity, neurogenesis, repetitive trans cranial magnetic stimulation, growth factors

Posted Date: May 26th, 2021

DOI: https://doi.org/10.21203/rs.3.rs-439386/v1

License: (c) (i) This work is licensed under a Creative Commons Attribution 4.0 International License. Read Full License 


\section{Abstract}

Background: The therapeutic benefits of repetitive trans cranial magnetic stimulation along with physical therapy to study the neuroplasticity and neurogenesis in ischemic stroke patients has not been fully elucidated. The aim of this study is to determine the neuroplasticity using serum growth factors as a surrogate marker, using $1 \mathrm{~Hz}$ rTMS with conventional physiotherapy in patients with sub acute ischemic stroke.

Methods: In this study, participants with first ever ischemic stroke $(\mathrm{N}=96)$, onset within 15 days were randomized after a run-in period of $75 \pm 7$ days along with standard physical therapy to receive 10 sessions of real $1 \mathrm{~Hz}$ rTMS $(\mathrm{N}=47)$ on contralesional premotor cortex or to sham stimulation ( $\mathrm{N}=49)$ for 2 weeks. Participants, investigators and outcome assessors were blinded. The primary efficacy outcome was change in the level of peripheral serum growth factors VEGF \& BDNF at third month. The secondary outcome was measurement of neurophysiological parameters and their correlation with growth factors levels.

Results: Modified intention to treat analysis showed significant up regulation in the mean level of serum VEGF \& BDNF from pre to post rTMS in Real rTMS Group. Trend of decrease in Resting Motor Threshold and increase in Motor Evoked Potential in the affected hand was seen. Statistically significant negative correlation between motor evoked potential and mean VEGF (ho $=-1.000, P<0.001$ ) in the affected hand in Real rTMS Group was seen.

Conclusion: Total ten sessions of $1 \mathrm{~Hz}$ rTMS plus physical therapy on contralateral hemisphere resulted in up regulation of serum growth factors possible reflecting improved neuroplasticity.

Trial funded by Indian Council of Medical Research (ICMR), India, CTRI/2016/02/006620. Retrospectively registered. Note: As this is not a regulatory trial, therefore under Indian regulatory authorities, ICMR, any trial registered before $1^{\text {st }}$ April 2018 was registered as retrospective study. Ethics committee approval for this study was taken in 2012.

\section{Background}

Stroke is the leading cause of adult neurological disability, affecting one in six people worldwide. Although various acute rehabilitative measures have been introduced to improve the outcome, but full recovery is often incomplete. Although reperfusion treatment benefits $20 \%$ of ischemic stroke patients, their effectiveness does not exceed $50-60 \%{ }^{1}$. Although various functional enhancing management techniques like physical therapy and conventional tools are available but till date no validated rehabilitative approach has been introduced which could restore the impaired functions by a complete recovery of the damaged tissue ${ }^{2}$

As new neuroprotective approaches are limited for clinicians and researchers, a need for new targets for the medium and long term prognosis of ischemic stroke is needed depending upon the size of infarction, speed and efficacy of the acute phase, therapeutic measures and the degree of neuro repair processes mediated by cerebral plasticity mechanism called cellular plasticity and its stimulation using growth factors causing neurogenesis, synaptogenesis mechanism and constitute a more translation therapeutic approach from the lab to the bedside ${ }^{1}$. Few supportive studies have evaluated the motor recovery pattern using non-invasive approach, despite of that more research in understanding the cellular mechanism and neuroprotective and restorative activities using trans cranial magnetic stimulation is needed ${ }^{3,4}$

To overcome this problem, neuroplasticity plays a vital role in the functional prognosis of stroke patients, and the same could be modulated by non-invasive neuro rehabilitation tool called trans cranial magnetic stimulation. This interactive mechanism between TMS and neurons produces wide range of electrophysiological, biochemical and molecular, cellular changes and also affects behavior, mood, memory, myelination and neuroplasticity ${ }^{5}$

At molecular level, certain neurotrophins like BDNF, VEGF are found to be upregulated in the first 4 weeks after stroke, creating a " neuroplastic milieu" in which cortical remodeling within the intact brain is optimized ${ }^{6}$

Various animal studies have studied the post ischemic molecular events in which growth of new synapses and dendritic branching modifies the intact sensorimotor cortex and subcortical structures contributing to the recovery of cognitive and motor performance, but understanding the neuroplasticity and neurogenesis model in patients with ischemic stroke recovery is yet to be studied ${ }^{7,8,9}$. Few researchers have studied the reduction in the oxidative stress and enhancement in the proliferation and signaling pathways using extremely low magnetic field with physical rehabilitation with few endothelial biomarkers in acute ischemic stroke patients ${ }^{10}$ but understanding the neuro pathophysiology and neurogenesis during Ischemic Stroke recovery stage is still lacking.

Therefore, the aim of this study was to assess the serum growth factors in studying the cortical excitability measured through neurophysiological parameters by administrating low frequency rTMS as a non-invasive stimulation approach in combination with conventional physical therapy in sub acute ischemic stroke patients.

\section{Methods}

The study methodology protocol was considered and followed from our previous published article ${ }^{11}$, except the growth factor estimation and neurophysiological outcomes which is the secondary objective of our research in this manuscript.

\section{Participants, Setting and Study Design}

The study was conducted at the All India Institute of Medical Sciences (AllMS), New Delhi. This was a single center, randomized, parallel group, double blind, sham controlled trial. AlIMS Ethics committee approved the study protocol. The trial was registered at the Clinical Trial Registry India (CTRI/2016/02/006620) 
and funded by Indian Council of Medical Research (ICMR), India. All the participants or their caregivers gave written informed consent. We included patients aged 18-75 years, with first ever-acute ischemic stroke, within last 15 days documented by CT/MRI scan of the head and NIHSS of 4-20. We excluded participants who were medically unstable, pregnant, with any other brain lesion (tumor, infection), comatose or on mechanical ventilation, history of epilepsy or having any surgical implant/ pacemaker.

\section{Procedure}

\section{Pre-randomization run-in period}

A trained licensed physiotherapist provided the standard physical therapy to all recruited participants. All participants received a standard care, which includes passive, active and active assisted exercises. Participants were encouraged to continue motor training at home after discharge and total number of hours of an individual exercise was monitored.

\section{Growth factor estimation}

Approximately $5 \mathrm{ml}$ non- fasting intravenous blood sample was withdrawn in an EDTA vial for the estimation of serum VEGF (pg/ml) and BDNF ( $\mathrm{ng} / \mathrm{ml}$ ) at the time of recruitment, pre-rTMS and post rTMS period. Samples were then immediately centrifuged $\left(1500 \mathrm{~g} / 15 \mathrm{minutes}\right.$, and serum was stored at $-70^{\circ} \mathrm{C}$ until assayed. Both VEGF $(\mathrm{pg} / \mathrm{ml})$ and BDNF $(\mathrm{ng} / \mathrm{ml})$ levels were measured using standard quantitative sandwich ELISA (Quantikine, USA) kits obtained from R \& D Systems. Samples from each individual were analyzed in a triplicate and subsequently used in all further statistical analysis.

\section{Randomization}

Participants were randomly assigned in a 1:1 ratio to receive either Real rTMS or Sham rTMS. Randomization was done using a computer-generated randomization sequence. Allocations were concealed using sealed, opaque, numbered envelopes and were opened only during the time of randomization phase. The investigator, physiotherapist and participants were masked to the treatment allocation. Lab technician who was not part of the study was responsible for the random allocation of participants to Sham or Real rTMS Group. The outcome assessor was also blinded to allocation.

\section{Interventions}

Low frequency rTMS was performed using Magstim Rapid stimulator, Magstim Ltd, UK equipped with air-cooled figure of eight coil (70 mm) i:e biphasic pulse. The resting motor evoked potential (MEP) was ascertained using an electromyogram, recording from the Abductor Pollicis Brevis (APB) in keeping with the International Federation of Clinical Neurophysiology (IFCN) recommendations ${ }^{12}$. The coil was placed tangentially to the scalp over the hand area of the primary motor cortex to calculate hot spot. Hot spot was defined as the location on the scalp where stimulation of a slightly supra threshold intensity elicited the largest motor evoked potential (MEP) in the APB muscle. After the hot spot was identified, resting motor threshold was determined using the lowest stimulus intensity to produce motor evoked potential of $>50 \mu \mathrm{V}$ peak-to-peak amplitude in 5 out of 10 subsequent trails. If no MEP was obtained at the time of hot spot calculation in the affected ipsilesion M1, then the hotspot was defined as the symmetric location to the contra lesion M1.The stimulation parameters were chosen in accordance with the safety guidelines for TMMS $^{12}$

Total 750 pulses, 75 trains using low frequency $(1 \mathrm{~Hz})$ with inter train interval of 45 seconds at calculated intensity of $110 \%$ resting motor threshold (RMT) (Fc3/Fc4), was administered to the randomized patient. Localization was done using $10-20$ EEG methods. Sham rTMS pulses were administered using the same stimulation parameters over the contra-lesion premotor cortex area with the figure of eight coil angled at 90 degrees from the scalp. Patient was aroused throughout the rTMS administration.

The rTMS sessions on each day was followed by 45-minute conventional physical therapy regime given by a trained physiotherapist.

\section{Monitoring for complications}

All participants were monitored for any adverse events. A checklist of previously reported side effects was used to report any possible adverse events.

\section{Outcomes}

The co-primary efficacy outcomes were changes in serum growth factors level VEGF ( $\mathrm{pg} / \mathrm{ml}), \mathrm{BDNF}(\mathrm{ng} / \mathrm{ml})$ measured at 3 months of stroke onset. The secondary efficacy outcomes were change in the physiological markers Resting Motor Threshold, Motor Evoked Potential and change in the MEP duration during rest at 3 months stroke onset.

\section{Sample size calculation}

Sample size was calculated for primary clinical efficacy outcome, $\mathrm{mBI}$ with a superiority hypothesis assumption. Calculation was based on the study done by Khedr et. al. ${ }^{13}$ who reported $34.6 \%$ and $7.7 \%$ of good/excellent outcome under rTMS and sham rTMS respectively, which yielded a sample size of 45 in each arm ( $90 \%$ power and a two-sided a level of $5 \%$ ). Adjusting for $10 \%$ attrition we estimated a sample size of 100 (50 in each arm)

\section{Statistical analysis}

Statistical analysis was performed using STATA version 14.1 and was based on modified intention to treat principle. Normal distribution was tested using Kolmogorov - Smirnov statistic. Study data was not following normal distribution; hence non-parametric tests were applied. For Categorical data, $2 \times 2$ table was generated. Chi-square test/ Fisher's exact test was applied to compare the properties in the two groups. Between groups comparisons were carried out 
using Wilcoxon's rank sum test. Wilcoxon signed rank test was used to assess changes in the scores within the same group. Non-parametric independent test analysis was also done using log algorithm. Nemar test was applied for the categorical variable. For serum estimation, non- parametric independent t-test was applied. For correlation analysis, spearman correlation was applied.

\section{Rationale}

The rationale of the study was to conduct the clinical translation research using rTMS along with physiotherapy at sub-acute recovery stage which is considered as the golden period for the initiation of exogenous restorative therapies. Endogenous repair reaches peak levels at this stage followed by the functional reorganization and enhances neural plasticity in the brain ${ }^{14}$.

\section{Results}

Between August 2012 and February 2016, 445 participants with acute ischemic stroke were screened and 139 participants fulfilling the eligibility criteria were recruited into the pre- rTMS run-in phase. During the run-in period, 35 participants withdrew consent and 4 died after discharge from the hospital. Randomization was done in 100 participants (Fig. 1). Four participants were excluded after randomization (consent withdrawn after randomization). The baseline characteristics were comparable between Real rTMS $(n=47)$ and sham rTMS $(n=49)$. Mean age in Real rTMS arm was $54.85 \pm 13.39$ vs. $52.89 \pm$ 14.95 years in Sham rTMS arm. There were more of male compared to female in both the groups (Table 1.). Mean serum biomarkers VEGF (pg/ml) and BDNF $(\mathrm{ng} / \mathrm{ml})$ were comparable. All study participants received standard of care and at least one session of real or sham rTMS. Seven participants in real rTMS were lost to follow up. One participant had seizure and un-blinding was done for that participant and was managed symptomatically. Modified intention to treat analysis was done for 47 participants in real and 49 participants in sham rTMS arm.

Growth Factors Estimation:

Compared to healthy controls, down regulation expression level of mean serum BDNF was seen. On the other hand, mean VEGF was elevated on $5^{\text {th }}$ day at the time of recruitment. (Table 2. \& 3. \& Fig. 4 \& 5).

Non-parametric independent t-test showed significant increase in the mean VEGF (pg/ml) level in Real rTMS Group 797.24 \pm 224.27 vs. $637.04 \pm 193.28$, $\mathrm{p}<0.001$ (Fig. 2.). Statistically significant change in the magnitude i:e delta level of VEGF (pg/ml), $-68.83 \pm 245.65)$ was higher in the Real rTMS arm vs. 48.32 \pm 298.10 in Sham rTMS arm.

Statistical significant increase in the mean level of serum BDNF (pg/ml) was seen in Real rTMS Group, $30.64 \pm 9.64$ vs. Sham r TMS, $25.12 \pm 10.11$, p<0.001(Fig.3.)

Neurophysiological Markers:

Resting Motor Threshold: -

Non-parametric independent t-test showed a trend of decrease in the RMT in the affected hand in the Real rTMS Group $69.40 \pm 22.34$ vs. $74.35 \pm 14.72$ in Sham rTMS Group, $P=0.31$. Compared to the control arm, total $N=14$ patients have shown change in the motor threshold level in active arm whereas a vice versa effect with an increase in the Resting Motor Threshold in the unaffected hand was seen in the Real TMS Group $76.17 \pm 13.09$ vs. $73.64 \pm 16.00, P=0.35$ in Sham rTMS Group. Compared to the control arm, total $N=23$ patients in Real rTMS showed change in the motor threshold level (Table 4. \& 5.)

\section{Motor Evoked Potential: -}

Non parametric independent t-test analysis showed a decreased in the motor evoked potential amplitude in the non - affected hand in Real TMS Group 189.66 \pm 77.64 Vs. Sham rTMS Group which was $158.17 \pm 82.82, P=0.22$ whereas an increase in the MEP in the affected hand was seen in both the Groups immediate after rTMS intervention, $\mathrm{P}=0.83$ (Table 6.)

\section{Absolute change in the MEP duration:-}

Significant increase in the mean absolute change in the MEP duration (in milli seconds) in the affected hand was seen in the Sham rTMS Group $7.44 \pm 2.58$ vs. $5.62 \pm 1.64$ in Real rTMS Group, P $<0.01$ at post rTMS, whereas no significant change in the unaffected hand was observed in either Group (Supplementary Table. 7)

\section{Correlation of growth factors vs. neurophysiological outcomes:}

\section{VEGF vs. Neurophysiological parameters:}

Spearman analysis showed significant negative correlation $(r=-1.000, P<0.001)$ of VEGF $(\mathrm{pg} / \mathrm{ml})$ with motor evoked potential in the affected hand in Real rTMS Group, $N=05$ compared to Sham rTMS Group $(r=0.2822, P=0.37), N=12$ (Table 8. \& 9.)

\section{BDNF vs. Neurophysiological parameters:}

Spearman correlation showed statistically positive correlation $(r=0.9000, P=0.03)$ of BDNF $(\mathrm{ng} / \mathrm{ml})$ with delta MEP duration in the unaffected hand in Real rTMS Group vs. Sham Group ( $r=-0.2465, P=0.43$ ) (Table 10. \& 11.)

\section{Adverse events}


One participant in the real TMS arm developed seizure (generalized tonic clonic seizure) 18 hours after the fourth session and about $1 \mathrm{~h}$ after waking from sleep in the morning. CT scan of head did not reveal any fresh changes. He was treated with phenytoin and the event was reported to Institute's Ethics Committee. The participant did not receive any more TMS sessions. No other adverse events were reported in the participants.

\section{Discussion}

In this Randomized controlled study, we measured the levels of serum growth factors VEGF and BDNF after administrating low frequency rTMS onto the contra lesion hemisphere along with conventional physiotherapy in patients with sub acute ischemic stroke. We assessed the neurophysiological parameters as a secondary outcome. In addition to this, a correlation of growth factors levels with neurophysiological parameters was also done at third month.

Although various studies using human models have been conducted in understanding the motor and clinical recovery but very limited studies in humans have been done in understanding the cellular mechanisms mediating rTMS neuroprotective and restorative activities. We speculated that post stroke noninvasive treatment approach induced neurogenesis might play a future potential target for therapeutic intervention.

Post stroke acute recovery is known to be correlated with endothelial function. With the release of polypeptide growth factors after acute ischemic stroke, they are likely to play a vital role in the both cellular and molecular process underlying wound healing and functional recovery in Stroke patients. Various observational studies reveal that VEGF mRNA up regulates under hypoxic conditions and elevated levels at during 7th day and 3th month are found to be independently associated with good functional outcome ${ }^{15,1}$ In our study, we found significant change in the mean serum biomarkers VEGF and BDNF in an active arm i:e Real rTMS Group compared with placebo arm i.e. Sham rTMS Group with conventional physical therapy in Stroke patients at third month.

VEGF is the most prominent hypoxia inducible angiogenic factor and a key mediator of angiogenesis, which is an important process leading to reperfusion of ischemic brain tissue after acute stroke, and is also implicated as an essential factor promoting neurogenesis in the adult brain, increasing proliferation and differentiation of endothelial and neuronal progenitor cells ${ }^{16,17,18}$. Increased VEGF might have long term beneficial effects as a result of continued angiogenesis over several months ${ }^{15}$. Various studies have been conducted in understating the VEGF expression various stroke recovery stages. Study conducted by Sobrino et. al. ${ }^{1}$. had shown high serum VEGF level at day 7 and at 3 months which is directly associated with good functional outcome. Another study conducted by Lee and his colleagues ${ }^{19}$ showed positive correlation of serum VEGF with long term prognosis in patients with acute ischemia. Another study conducted by Baba and his team ${ }^{20}$ administered electrical stimulation for seven day in adult Wistar rats. Significant up regulation of both VEGF and BDNF was seen. Ours is the first study in assessing the role of rTMS using low frequency with conventional physical therapy arm using large sample size of ischemic stroke patients at third month. Compared to healthy controls, higher expression of mean VEGF level was seen at the time of recruitment, signifying good prognosis. Results in our study have shown significant up regulation of mean VEGF level in Real rTMS Group. Referring to our previous published study, rTMS intervention has shown significant improvement in the magnitude of functional outcome in the active TMS arm ${ }^{11}$. Although, no significant correlation of mean VEGF with functional outcome was seen but up regulation of mean VEGF at immediate rTMS could justify the role angiogenesis which might play an essential role in the pathophysiology of stroke recovery mediated by rTMS.

Another neurotropic factor called BDNF is known to be involved in ischemia induced neurogenesis processes, increases recruitment of endogenous progenitors to injured brain regions, mediating repair mechanisms and neuronal plasticity in various brain disorders. It also induces synaptogenesis, morphogenesis, and plasticity of dendritic spines, resulting in synapses with functionality 21,22 and also plays an important role as an effective indicator for rehabilitation interventions ${ }^{23}$. Supportive evidence based studies suggests that rTMS causes enhancement of peripheral BDNF levels in patients suffering from Depression. In healthy human subjects, BDNF has shown activation of BDNF signaling pathways ${ }^{24-26}$. There is accumulating evidence on the role of BDNF in therapeutic effects using low frequency rTMS. BDNF polymorphism (val66met) negatively influences the effect of rTMS on upper limb weakness. The changes in blood levels of BDNF may be due to TMS induced alteration of BDNF-TrkB signaling in the brain. Recent study conducted by Niimi et. al.,2016 27 investigated the molecular effects of rTMS using low frequency on serum levels of BDNF and other growth factors in stroke patients with upper limb hemiparesis. Statistically significant increase in the serum BDNF level was seen after two weeks combination therapy. A community based cohort study

${ }^{28}$ having $\mathrm{N}=3440$ participants was conducted in understanding the association of serum VEFG and BDNF levels \& based on their analysis it was concluded that lower serum BDNF and higher VEGF concentrations were associated with increased risk of incident.

Similar results could be seen in our study. Compared to healthy controls, lower levels of serum BDNF ( $\mathrm{ng} / \mathrm{ml}$ ) were expressed. Immediate post RTMS showed significant elevated levels of BDNF in Real rTMS Group. Similar findings were seen in an observational study conducted by Sobrino et. al., $2020^{1}$, which reveals that elevated levels of serum endothelial growth factors are associated with good functional outcome at 3 months' ischemic stroke onset.

Ours is the first randomized controlled study with larger sample size to evaluate the association of both serum VEGF and BDNF levels in correlation to noninvasive approach with routine physical therapy intervention in understating neuroplasticity in ischemic stroke patients.

Neurophysiological Markers:

The therapeutic effects of non-invasive stimulation i:e Trans cranial magnetic stimulation could only be evaluated by applying either low (inhibitory) on contra lesion or high (excitatory) on ipisilesion hemisphere in stroke patients. rTMS has been known to restore the unbalanced interhemispheric inhibition, which results in increased inhibition in the ischemic hemisphere and subsequent worsening in motor function whereas the neurophysiological effect of rTMS on neuronal activity has not been well explored. TMS is known to be used as an excellent non-invasive tool to map the electrophysiological changes in the cortical hemispheres. In addition, that, they are used to dissect physiological mechanisms underlying motor deficits, spontaneous motor recovery, and the effect of therapeutic interventions in ischemic stroke like motor threshold, motor evoked potential, latency period etc. 
Neurophysiological changes occur both in acute and sub acute phase of stroke mostly attributable to pathophysiological changes like reversal of diaschisis, resolution of edema etc. ${ }^{29}$. Neurophysiological parameters like MEP, amplitude, MEP duration could provide an important insight into the motor cortical function. ${ }^{30}$

As MEP plays a main dependent variable in understanding the cortical function, recently the role of MEP duration was recently studied in both resting and facilitated state using various stimulus intensities to understand the underlying physiological changes in MEP duration reflecting cortical and nonpropriospinal mechanism involvement in stroke recovery ${ }^{30}$

In our study, MEP duration was assessed during resting state at a calculated intensity of $110 \%$ RMT and was correlated with the change in serum growth factors level to determine the cortical mechanisms and ceiling effects. MEP duration was measured from MEP onset latency to the time at which the activity returned to baseline. We determined the absolute change in the MEP duration during rest. Compared to control arm, statistically significant positive correlation of serum BDNF with delta MEP duration in the unaffected hand was seen in the active arm. Ours is the first study to correlate serum growth factor with change in the MEP duration in sub acute ischemic stroke patients using TMS as a main dependent factor in understanding the ceiling effect. Marisa and her colleagues measured an increase in duration of MEP during contraction in a group of healthy volunteers and on a group of patients with various neurological disorders. Compared to healthy controls, significant difference in the MEP duration at rest was seen ${ }^{31}$

An observational study was conducted to understand the physiological process underlying changes in MEP duration using TMS on healthy controls. Significant correlation of cortical inhibition and MEP duration was seen which might contribute as a future biomarker of motor cortical function ${ }^{30}$

Recent meta-analysis conducted by Zhang et. al., $2017^{32}$ reported that low frequency rTMS as an add on therapy improves functional recovery in Stroke patients. Trend of decrease in the cortico excitability, reflected in the form of Resting Motor Threshold and increase in the Motor Evoked Potential Amplitude in the affected hemisphere and vice versa has been found to be associated with motor recovery ${ }^{33}$. The pooled effects could also be seen in other supportive evidences ${ }^{34,35,36}$ Our results are consistent with the study done by Du and his team ${ }^{37}$, in which significant behavior neurophysiological correlation between corticospinal excitability and motor improvement was seen at 3 months that indicates that enhanced motor cortical excitability in the stroke hemisphere is an important precondition for neural plasticity, which may allow the surviving neurons to reorganize in response to therapy. A trend of cortico excitability was seen in the affected arm in the neurophysiological parameters with a decrease in Resting Motor Threshold, increase in the Motor Evoked Potential in Real rTMS Group.

\section{Conclusions}

The primary and secondary outcomes of the study have supported our study hypothesis. Higher levels of serum endothelial growth factors and significant change in the neurophysiological markers and its significant correlation with growth factors might play a vital role in understanding the neuroplasticity mechanism in stroke recovery. Follow up randomized controlled studies with large sample size are required to understand the role of TMS intensity and its exhibited ceiling effect in ischemic stroke recovery.

\section{Abbreviations}

rTMS: repetitive trans cranial magnetic stimulation

TMS: Trans cranial Magnetic Stimulation

BDNF: Brain derived neurotrophic factor

VEGF: Vascular endothelial growth factor

AllMS : All India Institute of Medical Sciences

ICMR : Indian Council of Medical Research

RMT: Resting Motor Threshold

MEP: Motor Evoked Potential

APB: Abductor Pollicis Brevis muscle

mBl: modified Barthel Index

CT : Computerized Tomography

CONSORT : Consolidated Standards of Reporting Trials

t-PA : recombinant tissue Plasminogen Activator

IFCN : International Federation of Clinical Neurophysiology

MT : Motor Threshold 
Unaff. : Unaffected

Aff. : Affected

\section{Declarations}

\section{Acknowledgements}

My heartfelt thanks to my parents for their unconditional support. My especial thanks to my co-investigators and colleagues for their invaluable guidance and support over the years. Finally, I would like to thank my mentor Dr. Padma Srivastava for supporting me, keeping an unconditional trust on me and helping me during the difficult period of my research, and making our research successful.

\section{Funding}

This study was funded by the Indian central government funding agency Indian Council of Medical Research, New Delhi, Trial Registration number: CTRI Number: CTRI/2016/02/006620

\section{Availability of data and materials}

The datasets generated and/or analysed during the current study are not publicly available due heavy size of data excel sheet but are available from the corresponding author on a reasonable request.

\section{Ethics approval and consent to participate :}

Ethical approval was taken from the Institute's Ethics Committee and as this was a Randomized Controlled Trial, so informed consent was taken. Informed consent was taken from all the participants in the study.

\section{Consent for Publication}

Consent and approval for manuscript publication was taken from all the respective co-author's of this study

\section{Competing Interest}

The authors declare that they have no competing interest.

\section{Author's Contributions}

HS: prepared the manuscript, acquired the entire research data; NK: assisted in designing rTMS protocol and neurophysiological parameters; RM: helped in growth factor analysis; SV: analysis and interpretation of the data; MV: defined the study design, clinical recruitment, funding and IRB approval, revising the manuscript, critically for important intellectual content. All authors read and approved the final manuscript.

\section{References}

1. Sobrino T, Rodríguez-Yáñez M, Campos F, et al. Association of High Serum Levels of Growth Factors with Good Outcome in Ischemic Stroke: a Multicenter Study. Trans/ Stroke Res. 2020. doi:10.1007/s12975-019-00747-2

2. Fisicaro F, Lanza G, Grasso AA, et al. Repetitive transcranial magnetic stimulation in stroke rehabilitation: review of the current evidence and pitfalls. Ther Adv Neurol Disord. 2019. doi:10.1177/1756286419878317

3. Hsu WY, Cheng CH, Liao KK, Lee IH, Lin YY. Effects of repetitive transcranial magnetic stimulation on motor functions in patients with stroke: A metaanalysis. Stroke. 2012;43(7):1849-1857. doi:10.1161/STROKEAHA.111.649756

4. Le Q, Qu Y, Tao Y, Zhu S. Effects of repetitive transcranial magnetic stimulation on hand function recovery and excitability of the motor cortex after stroke: A meta-analysis. Am J Phys Med Rehabil. 2014. doi:10.1097/PHM.0000000000000027

5. León Ruiz M, Rodríguez Sarasa ML, Sanjuán Rodríguez L, Benito-León J, García-Albea Ristol E, Arce Arce S. Current evidence on transcranial magnetic stimulation and its potential usefulness in post-stroke neurorehabilitation: Opening new doors to the treatment of cerebrovascular disease. Neurologia. 2018. doi:10.1016/j.nrl.2016.03.008

6. Ploughman M, Austin MW, Glynn L, Corbett D. The Effects of Poststroke Aerobic Exercise on Neuroplasticity: A Systematic Review of Animal and Clinical Studies. Trans/ Stroke Res. 2014;6(1):13-28. doi:10.1007/s12975-014-0357-7

7. Nudo RJ. Recovery after brain injury: Mechanisms and principles. Front Hum Neurosci. 2013. doi:10.3389/fnhum.2013.00887

8. Chan SJ, Love C, Spector M, Cool SM, Nurcombe V, Lo EH. Endogenous regeneration: Engineering growth factors for stroke. Neurochem Int. 2017;107:5765. doi:10.1016/j.neuint.2017.03.024

9. Huang X, Liu Y, Bai S, Peng L, Zhang B, Lu H. Granulocyte colony stimulating factor therapy for stroke: A pairwise meta-analysis of randomized controlled trial. PLoS One. 2017. doi:10.1371/journal.pone.0175774

10. Cichoń N, Bijak M, Czarny P, et al. Increase in blood levels of growth factors involved in the neuroplasticity process by using an extremely low frequency electromagnetic field in post-stroke patients. Front Aging Neurosci. 2018. doi:10.3389/fnagi.2018.00294 
11. Sharma H, Vishnu VY, Kumar N, et al. Efficacy of Low-Frequency Repetitive Transcranial Magnetic Stimulation in Ischemic Stroke: A Double-Blind Randomized Controlled Trial. Arch Rehabil Res Clin Transl. 2020;2(1):100039. doi:10.1016/j.arrct.2020.100039

12. Rossi S, Hallett M, Rossini PM, et al. Safety, ethical considerations, and application guidelines for the use of transcranial magnetic stimulation in clinical practice and research. Clin Neurophysiol. 2009;120(12):2008-2039. doi:10.1016/j.clinph.2009.08.016

13. Khedr EM. Therapeutic trial of repetitive transcranial magnetic stimulation after acute ischemic stroke. Neurology. 2005;65:466-468. http://dx.doi.org/10.1212/01.wnl.0000173067.84247.36.

14. Cramer SC. Repairing the human brain after stroke: I. Mechanisms of spontaneous recovery. Ann Neurol. 2008;63(3):272-287. doi:10.1002/ana.21393

15. Slevin M, Krupinski J, Slowik a., Kumar P, Szczudlik a., Gaffney J. Serial Measurement of Vascular Endothelial Growth Factor and Transforming Growth Factor- 1 in Serum of Patients With Acute Ischemic Stroke. Stroke. 2000;31(8):1863-1870. doi:10.1161/01.STR.31.8.1863

16. Sobrino T, Pérez-Mato M, Brea D, Rodríguez-Yáñez M, Blanco M, Castillo J. Temporal profile of molecular signatures associated with circulating endothelial progenitor cells in human ischemic stroke. J Neurosci Res. 2012. doi:10.1002/jnr.23068

17. Sun Y, Jin K, Xie L, et al. VEGF-induced neuroprotection, neurogenesis, and angiogenesis after focal cerebral ischemia. J Clin Invest. 2003. doi:10.1172/jci17977

18. Heissig B, Hattori K, Dias S, et al. Recruitment of stem and progenitor cells from the bone marrow niche requires MMP-9 mediated release of Kit-ligand. Cell. 2002. doi:10.1016/S0092-8674(02)00754-7

19. Lee S, Lee K, Kim Y, Kim SH, Koh S, Lee YJ. Serum VEGF levels in acute ischaemic strokes are correlated with long-term prognosis. 2010:45-51. doi:10.1111/j.1468-1331.2009.02731.x

20. Baba T, Kameda M, Yasuhara T, et al. Electrical stimulation of the cerebral cortex exerts antiapoptotic, angiogenic, and anti-inflammatory effects in ischemic stroke rats through phosphoinositide 3-kinase/akt signaling pathway. Stroke. 2009. doi:10.1161/STROKEAHA.109.563627

21. Alsina B, Vu T, Cohen-Cory S. Visualizing synapse formation in arborizing optic axons in vivo: Dynamics and modulation by BDNF. Nat Neurosci. 2001. doi:10.1038/nn735

22. Lessmann V, Gottmann K, Malcangio M. Neurotrophin secretion: Current facts and future prospects. Prog Neurobiol. 2003. doi:10.1016/S03010082(03)00019-4

23. Qiao H-J, Li Z-Z, Wang L-M, Sun W, Yu J-C, Wang B. Association of lower serum Brain-derived neurotrophic factor levels with larger infarct volumes in acute ischemic stroke. J Neuroimmunol. 2017;307:69-73. doi:10.1016/j.jneuroim.2017.04.002

24. Yukimasa T, Tamagawa A, Uozumi T, et al. High-frequency repetitive transcranial magnetic stimulation improves refractory depression by influencing catecholamine and brain-derived neurotrophic factors. Pharmacopsychiatry. 2006. doi:10.1055/s-2006-931542

25. Zanardini R, Gazzoli A, Ventriglia M, et al. Effect of repetitive transcranial magnetic stimulation on serum brain derived neurotrophic factor in drug resistant depressed patients. J Affect Disord. 2006. doi:10.1016/j.jad.2005.12.029

26. Wang HY, Crupi D, Liu J, et al. Repetitive transcranial magnetic stimulation enhances BDNF-TrkB signaling in both brain and lymphocyte. J Neurosci. 2011. doi:10.1523/JNEUROSCI.2125-11.2011

27. Niimi M, Hashimoto K, Kakuda W, et al. Role of brain-derived neurotrophic factor in beneficial effects of repetitive transcranial magnetic stimulation for upper limb hemiparesis after stroke. PLoS One. 2016;11(3). doi:10.1371/journal.pone.0152241

28. Pikula A, Beiser AS, Chen TC, et al. Serum Brain - Derived Neurotrophic Factor and Vascular. 2013. doi:10.1161/STROKEAHA.113.001447

29. Hendricks HT, Pasman JW, Merx JL, Van Limbeek J, Zwarts MJ. Analysis of recovery processes after stroke by means of transcranial magnetic stimulation. In: Journal of Clinical Neurophysiology. ; 2003. doi:10.1097/00004691-200305000-00004

30. Van Den Bos MAJ, Geevasinga N, Menon P, Burke D, Kiernan MC, Vucic S. Physiological processes influencing motor-evoked potential duration with voluntary contraction. J Neurophysiol. 2017. doi:10.1152/jn.00832.2016

31. Brum M, Cabib C, Valls-Solé J. Clinical value of the assessment of changes in MEP duration with voluntary contraction. Front Neurosci. 2016. doi:10.3389/fnins.2015.00505

32. Zhang L, Xing G, Shuai S, et al. Low-Frequency Repetitive Transcranial Magnetic Stimulation for Stroke-Induced Upper Limb Motor Deficit: A MetaAnalysis. Neural Plast. 2017. doi:10.1155/2017/2758097

33. Hallett M. Transcranial Magnetic Stimulation: A Primer. Neuron. 2007. doi:10.1016/j.neuron.2007.06.026

34. Fregni F, Boggio PS, Valle AC, et al. A sham-controlled trial of a 5-day course of repetitive transcranial magnetic stimulation of the unaffected hemisphere in stroke patients. Stroke. 2006;37(8):2115-2122. doi:10.1161/01.STR.0000231390.58967.6b

35. Sung WH, Wang CP, Chou CL, Chen YC, Chang YC, Tsai PY. Efficacy of coupling inhibitory and facilitatory repetitive transcranial magnetic stimulation to enhance motor recovery in hemiplegic stroke patients. Stroke. 2013;44(5):1375-1382. doi:10.1161/STROKEAHA.111.000522

36. Wang CP, Tsai PY, Yang TF, Yang KY, Wang CC. Differential Effect of Conditioning Sequences in Coupling Inhibitory/Facilitatory Repetitive Transcranial Magnetic Stimulation for PostStroke Motor Recovery. CNS Neurosci Ther. 2014. doi:10.1111/cns.12221

37. Du J, Tian L, Liu W, et al. Effects of repetitive transcranial magnetic stimulation on motor recovery and motor cortex excitability in patients with stroke: a randomized controlled trial. Eur J Neurol. 2016;23(11):1666-1672. doi:10.1111/ene.13105

\section{Tables}

Table.1 Showing Baseline characteristics between Real rTMS group, $N=47$ and Sham rTMS group, $N=49$ 


\begin{tabular}{|c|c|c|c|}
\hline \multirow[t]{4}{*}{ Variable } & Sham rTMS & Real rTMS & $\mathrm{P}$ \\
\hline & $N=49$ & $N=47$ & \\
\hline & Mean \pm S.D & Mean \pm S.D & \\
\hline & $95 \% \mathrm{Cl}$ & $95 \% \mathrm{Cl}$ & \\
\hline \multirow[t]{2}{*}{ Age } & $52.89 \pm 14.95$ & $54.85 \pm 13.39$ & 0.50 \\
\hline & $48.60-57.19$ & $50.91-58.78$ & \\
\hline \multicolumn{4}{|l|}{ Sex } \\
\hline Male & $34(69)$ & $33(77)$ & 0.93 \\
\hline Female & $15(31)$ & $14(30)$ & \\
\hline Hypertension & $32(65)$ & $35(74)$ & 0.60 \\
\hline Diabetes & $13(26)$ & $11(23)$ & 0.45 \\
\hline Smoking & $16(33)$ & $15(32)$ & 0.55 \\
\hline Tobacco Chewing & $6(12)$ & $4(8)$ & 0.39 \\
\hline IV-tPA & $6(12)$ & $6(12)$ & 0.59 \\
\hline \multicolumn{4}{|l|}{ Stroke Subtype } \\
\hline Large artery & $12(25)$ & $12(26)$ & \\
\hline Small artery & $4(8)$ & $3(6)$ & \\
\hline Lacunar & $2(4)$ & $1(2)$ & \\
\hline Cardio-embolic & $7(15)$ & $6(13)$ & \\
\hline Others & $24(48)$ & $25(53)$ & 0.98 \\
\hline \multicolumn{4}{|l|}{ Onset to enrollment } \\
\hline$\leq 7$ & $41(82)$ & 36 & \\
\hline \multirow[t]{2}{*}{$8-15$} & $6(12)$ & 11 & \\
\hline & $4.68 \pm 1.26$ & $4.96 \pm 1.56$ & 0.33 \\
\hline \multicolumn{4}{|l|}{ Onset to TMS } \\
\hline $60-75$ & $8(16)$ & $16(32)$ & \\
\hline \multirow[t]{2}{*}{$76-83$} & $39(78)$ & $31(62)$ & \\
\hline & $77.34 \pm 10.21$ & $77.51 \pm 5.38$ & 0.91 \\
\hline VEGF(pg/ml) & $721.37 \pm 217.2$ & $728.41 \pm 198.75$ & 0.09 \\
\hline BDNF(ng/ml) & $15.48 \pm 8.99$ & $18.73 \pm 10.79$ & 0.11 \\
\hline
\end{tabular}

Table 2. Table showing mean serum VEGF $(\mathrm{pg} / \mathrm{ml})$ level at various time points 


\begin{tabular}{|c|c|c|c|}
\hline & VEGF $(\mathrm{pg} / \mathrm{ml})$ & & \\
\hline \multirow[t]{3}{*}{ S. No. } & Real rTMS $n=47$ & Sham rTMS, $n=49$ & P Value \\
\hline & Mean \pm S.D & Mean \pm S.D & \\
\hline & Median (IQR) & Median (IQR) & \\
\hline \multirow[t]{2}{*}{ Baseline } & $728.41 \pm 198.75$ & $721.37 \pm 217.2$ & $0.9 *$ \\
\hline & $780(180)$ & $746.63(283.4)$ & \\
\hline \multirow[t]{2}{*}{ Pre- rTMS } & $637.23 \pm 187.65$ & $655.80 \pm 472.67$ & $0.1^{\star}$ \\
\hline & $658.2(207)$ & $579.6(476)$ & \\
\hline \multirow[t]{2}{*}{ Post rTMS } & $797.24 \pm 224.27$ & $673.04 \pm 193.28$ & $<0.001$ \\
\hline & $824(101)$ & $745(188)$ & \\
\hline \multirow[t]{2}{*}{ Healthy } & $435 \pm 275.12$ & & \\
\hline & 374 (357) & & \\
\hline \multirow[t]{2}{*}{ Delta VEGF = Base. - Post rTMS } & $-68.83 \pm 245.65$ & $48.32 \pm 298.10$ & $0.01 \#$ \\
\hline & $-34.40(179.6)$ & $87(242.37)$ & \\
\hline
\end{tabular}

$* \log$

\#Rank sum

Table 3. Table showing mean serum BDNF $(\mathrm{ng} / \mathrm{ml})$ level at various time points

\begin{tabular}{|c|c|c|c|}
\hline \multicolumn{4}{|c|}{ BDNF (ng/ml) } \\
\hline \multirow[t]{3}{*}{ S.No. } & Real rTMS n $=47$ & Sham rTMS, $n=49$ & P Value \\
\hline & Mean \pm S.D & Mean \pm S.D & \\
\hline & Median (IQR) & Median (IQR) & \\
\hline \multirow[t]{2}{*}{ Baseline } & $18.73 \pm 10.79$ & $15.48 \pm 8.99$ & 0.11 \\
\hline & $20.42(16.77)$ & $15.3(15.45)$ & \\
\hline \multirow{2}{*}{ Pre- rTMS } & $25.06 \pm 12.67$ & $22.73 \pm 6.94$ & 0.26 \\
\hline & $22.52(19.41)$ & $21.58(10.62)$ & \\
\hline \multirow[t]{2}{*}{ Post rTMS } & $30.64 \pm 9.64$ & $25.12 \pm 10.11$ & $<0.001$ \\
\hline & $31.95(10.50)$ & 24.64(11.94) & \\
\hline \multirow[t]{3}{*}{ Healthy } & $23.0 \pm 11.0$ & & \\
\hline & $20(14)$ & & \\
\hline & $N=50$ & & \\
\hline \multirow[t]{2}{*}{ BDNF delta = Base. - Post rTMS } & $-11.90 \pm 11.35$ & $-9.64 \pm 10.21$ & 0.33 \\
\hline & $-10.98(14.34)$ & $-8.21(11.82)$ & \\
\hline
\end{tabular}

Table 4. Table showing mean Resting Motor Threshold in affected hand from pre to post rTMS intervention

\begin{tabular}{|llll|}
\hline \multicolumn{3}{|l|}{ Resting Motor Threshold (RMT) Affected Hand } \\
\hline S. No. & Real rTMS & Sham rTMS & P Value \\
& Mean \pm S.D & Mean \pm S.D & \\
& Median (IQR) & Median (IQR) & \\
\hline Pre- rTMS & $93.30 \pm 9.33$ & $84.80 \pm 15.69$ & $0.11 \#$ \\
& $\mathrm{~N}=13$ & $\mathrm{~N}=21$ & \\
Post rTMS & $69.40 \pm 22.34$ & $74.35 \pm 14.72$ & 0.31 \\
& $\mathrm{~N}=27$ & $\mathrm{~N}=31$ & \\
\hline
\end{tabular}

\#rank sum 
Table 5. Table showing mean Resting Motor Threshold in non-affected hand from pre to post rTMS intervention

\begin{tabular}{|llll|}
\hline \multicolumn{3}{|l|}{ Resting Motor Threshold (RMT) Non-Affected Hand } \\
\hline S. No. & Real rTMS & Sham rTMS, $\mathrm{n}$ & P Value \\
& Mean \pm S.D & Mean \pm S.D & \\
\hline Pre- rTMS & $69.30 \pm 10.58$ & $70.92 \pm 11.79$ & 0.52 \\
& $\mathrm{~N}=40$ & $\mathrm{~N}=41$ & \\
Post rTMS & $76.17 \pm 13.09$ & $73.64 \pm 16.00$ & 0.59 \\
& $\mathrm{~N}=17$ & $\mathrm{~N}=25$ & \\
\hline
\end{tabular}

Table 6. Table showing mean Motor Evoked Potential in both affected hand from pre to post rTMS intervention

\begin{tabular}{|llll|}
\hline Motor Evoked Potential & Sham rTMS & Real rTMS & P- Value \\
Affected Hand & & & \\
Pre rTMS & $104.89 \pm 64.66$ & $93.43 \pm 77.16$ & 0.75 \\
Mean \pm SD & $\mathrm{N}=10$ & $\mathrm{~N}=6$ & \\
& & & \\
Post rTMS & $120.98 \pm 64.30$ & $116.64 \pm 58.16$ & 0.83 \\
Mean \pm SD & $\mathrm{N}=23$ & $\mathrm{~N}=16$ & \\
Non affected Hand & & & \\
Pre rTMS & & & \\
Mean \pm SD & $154.62 \pm 85.87$ & $193.93 \pm 93.32$ & $0.08^{\star}$ \\
& $\mathrm{N}=33$ & $\mathrm{~N}=33$ & \\
Post rTMS & & & \\
Mean \pm SD & $158.17 \pm 82.82$ & $189.66 \pm 77.64$ & 0.22 \\
& $\mathrm{~N}=25$ & $\mathrm{~N}=17$ & \\
\hline
\end{tabular}

${ }^{*} \log$ value

Table 7. Table showing mean absolute change in MEP duration in affected hand from pre to post rTMS intervention

\begin{tabular}{|llll|}
\hline Absolute change MEP duration (delta MEP duration) & Sham rTMS & Real rTMS & P- Value \\
Affected hand & & & \\
Pre rTMS & $5.9 \pm 2.37$ & $5.00 \pm 1.26$ & 0.40 \\
Mean \pm SD & $\mathrm{N}=10$ & $\mathrm{~N}=6$ & \\
& & & \\
& & & \\
Post rTMS & $7.44 \pm 2.58$ & $5.62 \pm 1.64$ & 0.01 \\
Mean \pm SD & $\mathrm{N}=23$ & $\mathrm{~N}=16$ & \\
Non affected Hand & & & \\
& & & \\
Pre rTMS & $7.14 \pm 2.60$ & $7.11 \pm 2.81$ & 0.96 \\
Mean \pm SD & $\mathrm{N}=34$ & $\mathrm{~N}=34$ & \\
& & & \\
Post rTMS & $7.91 \pm 3.34$ & $7.67 \pm 2.12$ & 0.79 \\
Mean \pm SD & $\mathrm{N}=24$ & $\mathrm{~N}=17$ & \\
\hline
\end{tabular}

Table 8. Table showing correlation between serum VEGF and neurophysiological markers from pre to post in Sham rTMS Group 


\begin{tabular}{|c|c|c|c|c|c|c|c|c|}
\hline \multicolumn{9}{|l|}{$\begin{array}{l}\text { Sham rTMS Group - } \\
\text { VEGF }(\mathrm{pg} / \mathrm{ml})\end{array}$} \\
\hline \multicolumn{2}{|l|}{ Variable } & $\begin{array}{l}\text { VEGF } \\
(\mathrm{pg} / \mathrm{ml})\end{array}$ & MT_post_unaff & MT_post_aff & MEP_post_unaff. & MEP_post_aff & Delta_MEPwidth_unaff. & Delta_MI \\
\hline \multirow[t]{2}{*}{ MT_post_unaff. } & rho & 0.2544 & 1.0000 & & & & & \\
\hline & $P$ & 0.4249 & & & & & & \\
\hline \multirow[t]{2}{*}{ MT_post_aff. } & rho & -0.0426 & -0.0283 & 1.0000 & & & & \\
\hline & $P$ & 0.8955 & 0.9305 & & & & & \\
\hline \multirow[t]{2}{*}{ MEP_post_unaff } & rho & -0.5221 & -0.1547 & -0.1129 & 1.0000 & & & \\
\hline & $\mathrm{P}$ & 0.0817 & 0.6313 & 0.7269 & & & & \\
\hline \multirow[t]{2}{*}{ MEP_post_aff } & rho & 0.2822 & 0.1125 & -0.2822 & 0.4246 & 1.0000 & & \\
\hline & $\mathrm{P}$ & 0.3742 & 0.7278 & 0.3742 & 0.1689 & & & \\
\hline \multirow[t]{2}{*}{ Delta_MEPduration_unaff. } & rho & -0.3298 & -0.6590 & 0.1454 & 0.5961 & 0.0847 & 1.0000 & \\
\hline & $P$ & 0.2952 & $0.0198^{*}$ & 0.6521 & 0.0408 & 0.7936 & & \\
\hline \multirow[t]{2}{*}{ Delta_MEPduration_aff. } & rho & -0.1137 & -0.3752 & -0.2842 & 0.5972 & 0.1661 & 0.6963 & 1.0000 \\
\hline & $\mathrm{P}$ & 0.7250 & 0.2294 & 0.3707 & 0.0403 & 0.6059 & 0.0119 & \\
\hline
\end{tabular}

Total observation, $\mathrm{N}=12$

Table 9. Table showing correlation between serum VEGF and neurophysiological markers from pre to post in Real rTMS Group rTMS intervention

\begin{tabular}{|c|c|c|c|c|c|c|c|c|}
\hline $\begin{array}{l}\text { Real rTMS Group - VEGF } \\
(\mathrm{pg} / \mathrm{ml})\end{array}$ & & & & & & & & \\
\hline Variable & & $\begin{array}{l}\text { VEGF } \\
(\mathrm{pg} / \mathrm{ml})\end{array}$ & MT_post_unaff. & MT_post_aff & MEP_post_unaff. & MEP_post_aff & Delta_MEPduration_unaff & Delta. \\
\hline \multirow[t]{2}{*}{ MT_post_unaff. } & rho & -0.2000 & 1.0000 & & & & & \\
\hline & $\mathrm{P}$ & 0.7471 & & & & & & \\
\hline \multirow[t]{2}{*}{ MT_post_aff. } & rho & 0.2000 & 0.8000 & 1.0000 & & & & \\
\hline & $\mathrm{P}$ & 0.7471 & 0.1041 & & & & & \\
\hline \multirow[t]{2}{*}{ MEP_post_unaff } & rho & -0.3000 & -0.3000 & -0.3000 & 1.0000 & & & \\
\hline & $\mathrm{P}$ & 0.6238 & 0.6238 & 0.6238 & & & & \\
\hline \multirow[t]{2}{*}{ MEP_post_aff } & rho & -1.0000 & 0.2000 & -0.2000 & 0.3000 & 1.0000 & & \\
\hline & $\mathrm{P}$ & 0.0000 & 0.7471 & 0.7471 & 0.6238 & & & \\
\hline \multirow[t]{2}{*}{ Delta_MEPduration_unafff } & rho & 0.6000 & -0.4000 & 0.1000 & 0.5000 & -0.6000 & 1.0000 & \\
\hline & $\mathrm{P}$ & 0.2848 & 0.5046 & 0.8729 & 0.3910 & 0.2848 & & \\
\hline \multirow[t]{2}{*}{ Delta_MEPduration_aff } & rho & 0.1026 & 0.4104 & 0.8208 & -0.0513 & -0.1026 & 0.3591 & 1.000 \\
\hline & $P$ & 0.8696 & 0.4925 & 0.0886 & 0.9347 & 0.8696 & 0.5528 & \\
\hline
\end{tabular}

Total observation, $\mathrm{N}=5$

Table 10. Table showing correlation between serum BDNF and neurophysiological markers from pre to post in Real rTMS Group 


\begin{tabular}{|c|c|c|c|c|c|c|c|c|}
\hline $\begin{array}{l}\text { Real rTMS Group - BDNF } \\
(\mathrm{ng} / \mathrm{ml})\end{array}$ & & & & & & & & \\
\hline Variable & & $\begin{array}{l}\text { BDNF } \\
(\mathrm{pg} / \mathrm{ml})\end{array}$ & MT_post_unaff. & MT_post_aff & MEP_post_unaff. & MEP_post_aff & Delta_MEPduration_unaff. & Delta_ \\
\hline \multirow[t]{2}{*}{ MT_post_unaff. } & rho & -0.3000 & 1.0000 & & & & & \\
\hline & $P$ & 0.6238 & & & & & & \\
\hline \multirow[t]{2}{*}{ MT_post_aff. } & rho & 0.0000 & 0.8000 & 1.0000 & & & & \\
\hline & $\mathrm{P}$ & 1.000 & 0.1041 & & & & & \\
\hline \multirow[t]{2}{*}{ MEP_post_unaff } & rho & 0.8000 & -0.3000 & -0.3000 & 1.0000 & & & \\
\hline & $\mathrm{P}$ & 0.1041 & 0.6238 & 0.6238 & & & & \\
\hline \multirow[t]{2}{*}{ MEP_post_aff } & rho & -0.3000 & 0.2000 & -0.2000 & 0.3000 & 1.0000 & & \\
\hline & $\mathrm{P}$ & 0.6238 & 0.7471 & 0.7471 & 0.6238 & & & \\
\hline \multirow[t]{2}{*}{ Delta_MEPduration_unaff } & rho & 0.9000 & 0.4000 & 0.1000 & 0.5000 & -0.6000 & 1.0000 & \\
\hline & $\mathrm{P}$ & 0.0374 & 0.5046 & 0.8729 & 0.3910 & 0.2848 & & \\
\hline \multirow[t]{2}{*}{ Delta_MEPduration_aff } & rho & 0.2052 & 0.4104 & 0.8208 & -0.0513 & -0.1026 & 0.3591 & 1.000 \\
\hline & $P$ & 0.7406 & 0.4925 & 0.0886 & 0.9347 & 0.8696 & 0.5528 & \\
\hline
\end{tabular}

Total Observation, $\mathrm{N}=05$

Table 11. Table showing correlation between serum BDNF and neurophysiological markers from pre to post in Sham rTMS Group

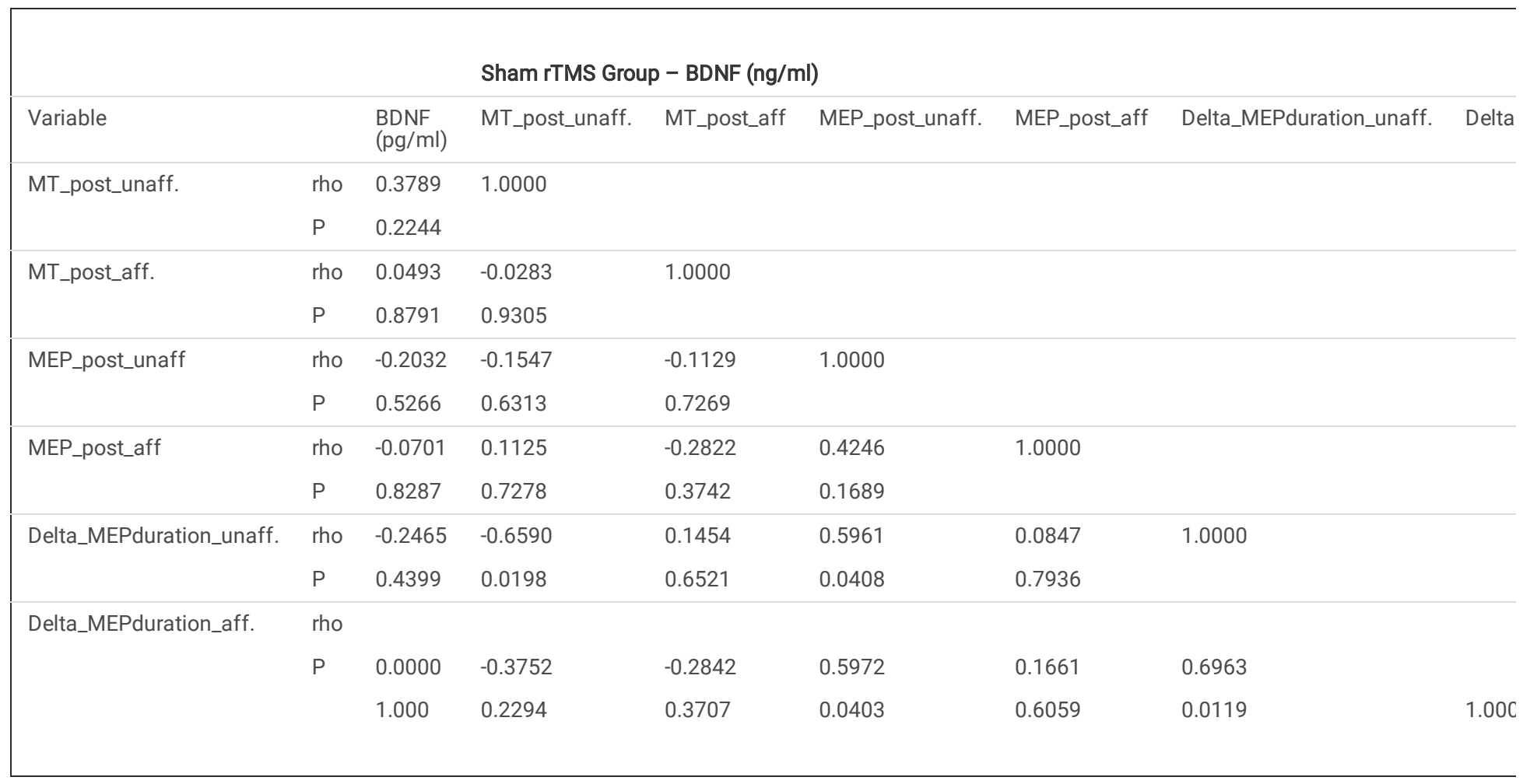

Total observation, $\mathrm{N}=12$

\section{Figures}




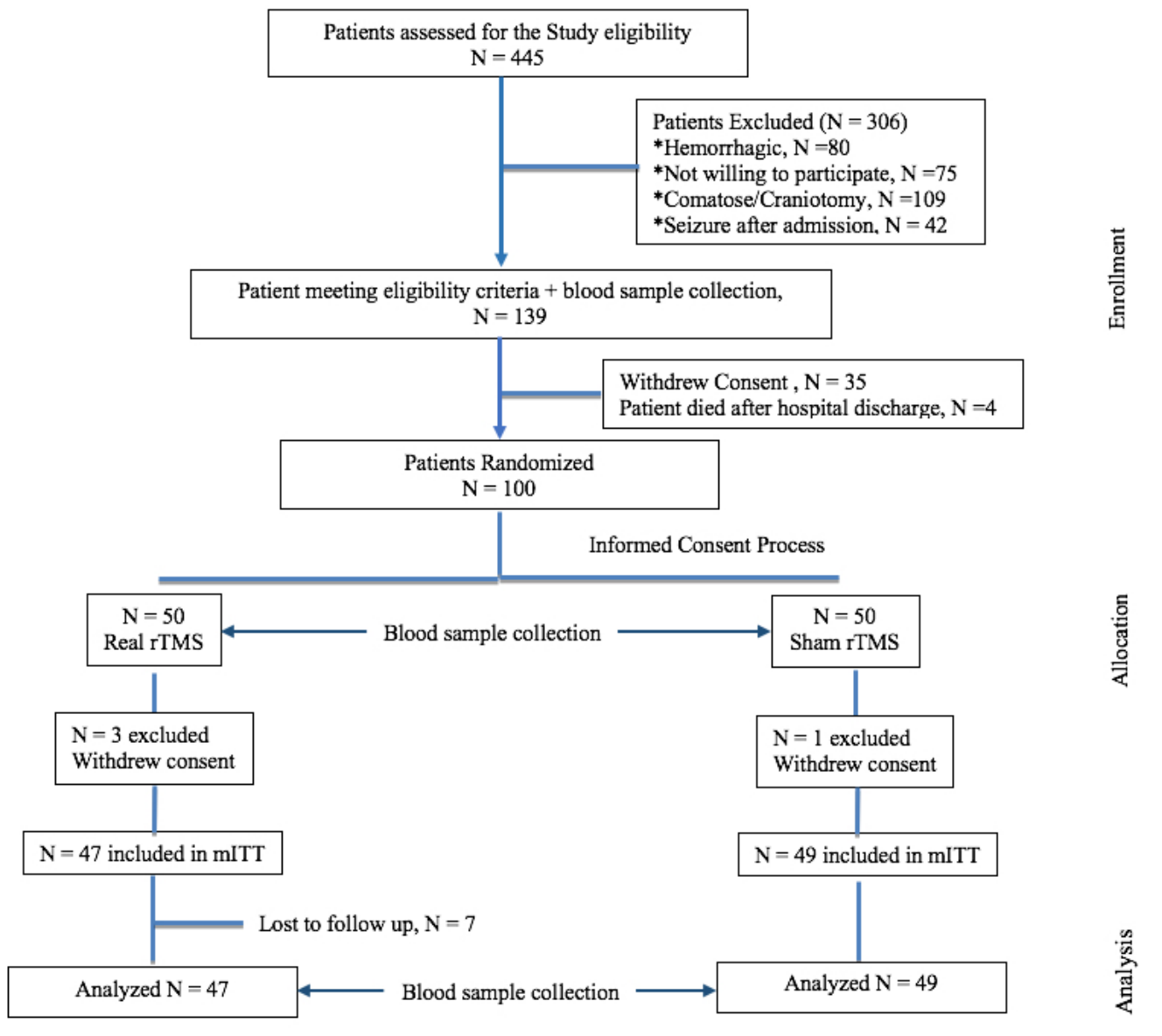

Figure 1

CONSORT flow diagram of the study

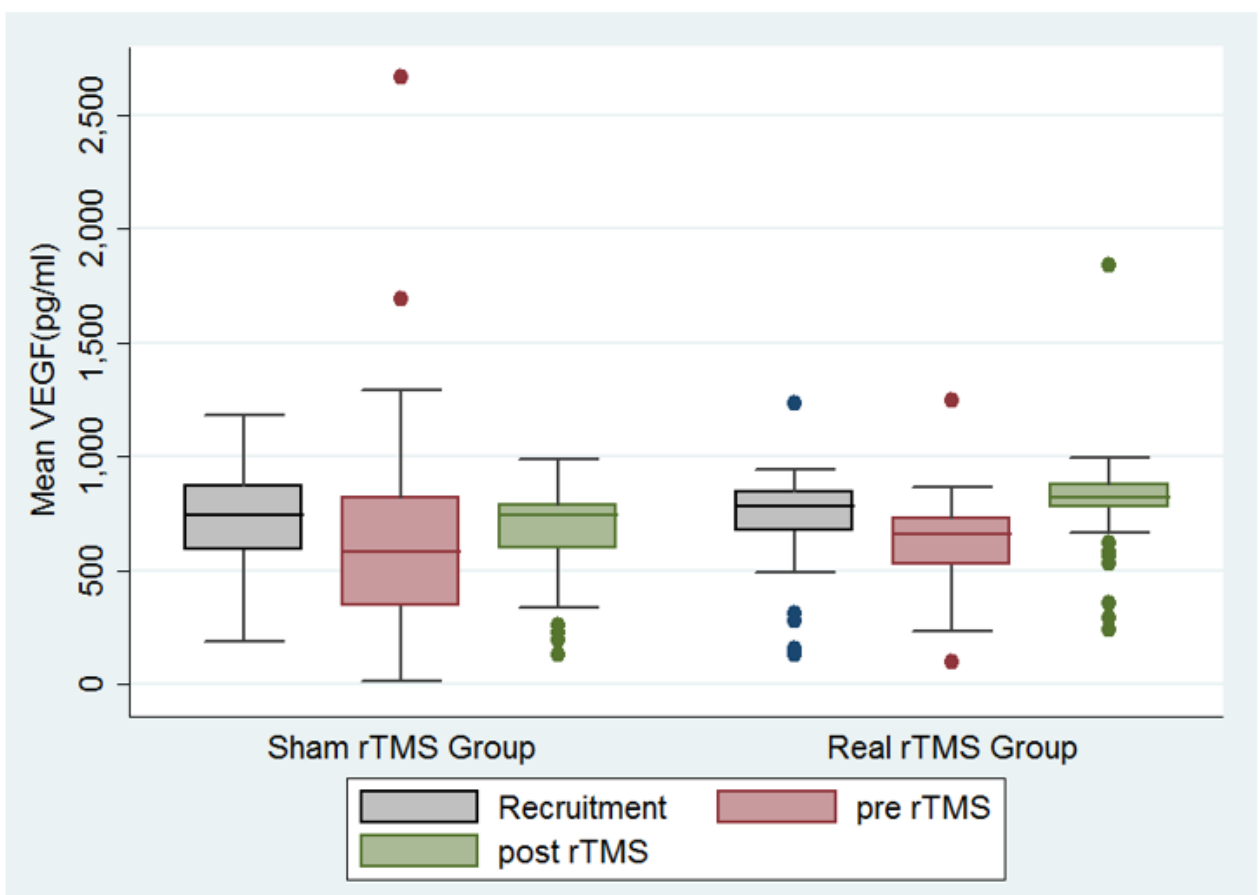

Figure 2

Whisker plot showing median distribution of serum VEGF $(\mathrm{pg} / \mathrm{ml})$ change from pre to post rTMS between Real rTMS Group, N= 47 and Sham rTMS Group ,N = 49

Page 14/16 


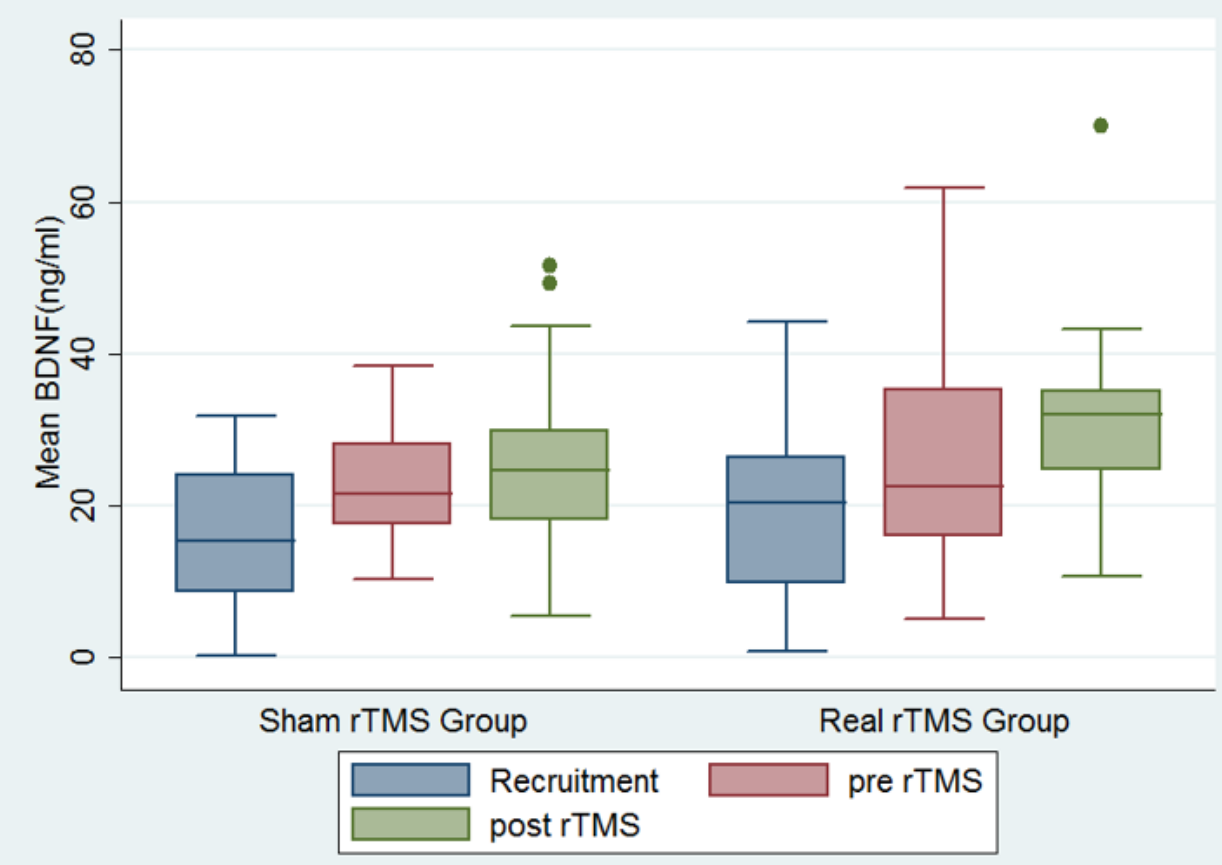

Figure 3

Whisker plot showing median distribution of serum BDNF $(\mathrm{ng} / \mathrm{ml})$ change from pre to post rTMS between Real rTMS Group, N= 47 and Sham rTMS Group , N $=49$

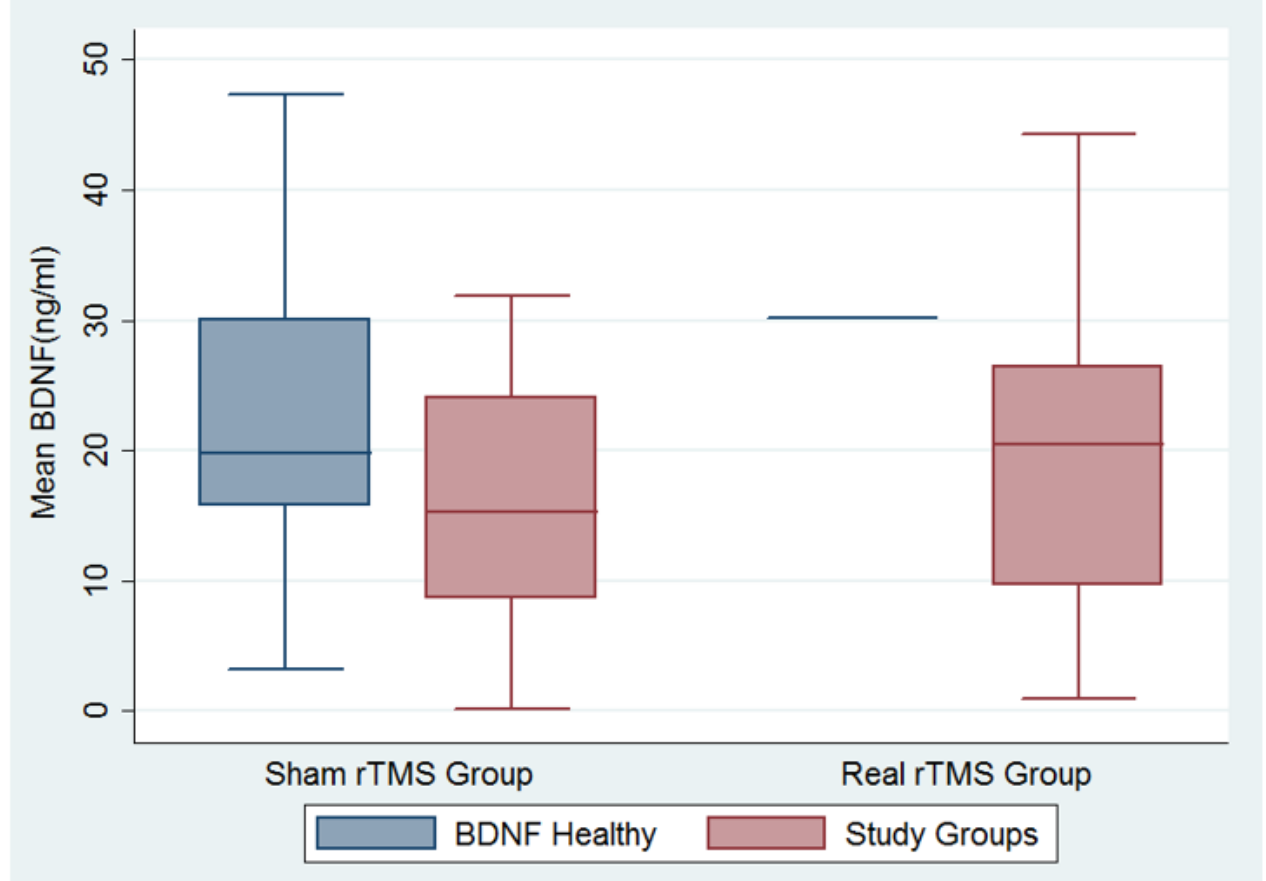

Figure 4

Whisker plot showing median serum BDNF $(\mathrm{ng} / \mathrm{ml})$ change at recruitment between the Sham rTMS Group, $\mathrm{N}=50$, Real rTMS Group, $\mathrm{N}=50$ \& Healthy volunteers, $\mathrm{N}=50$ 


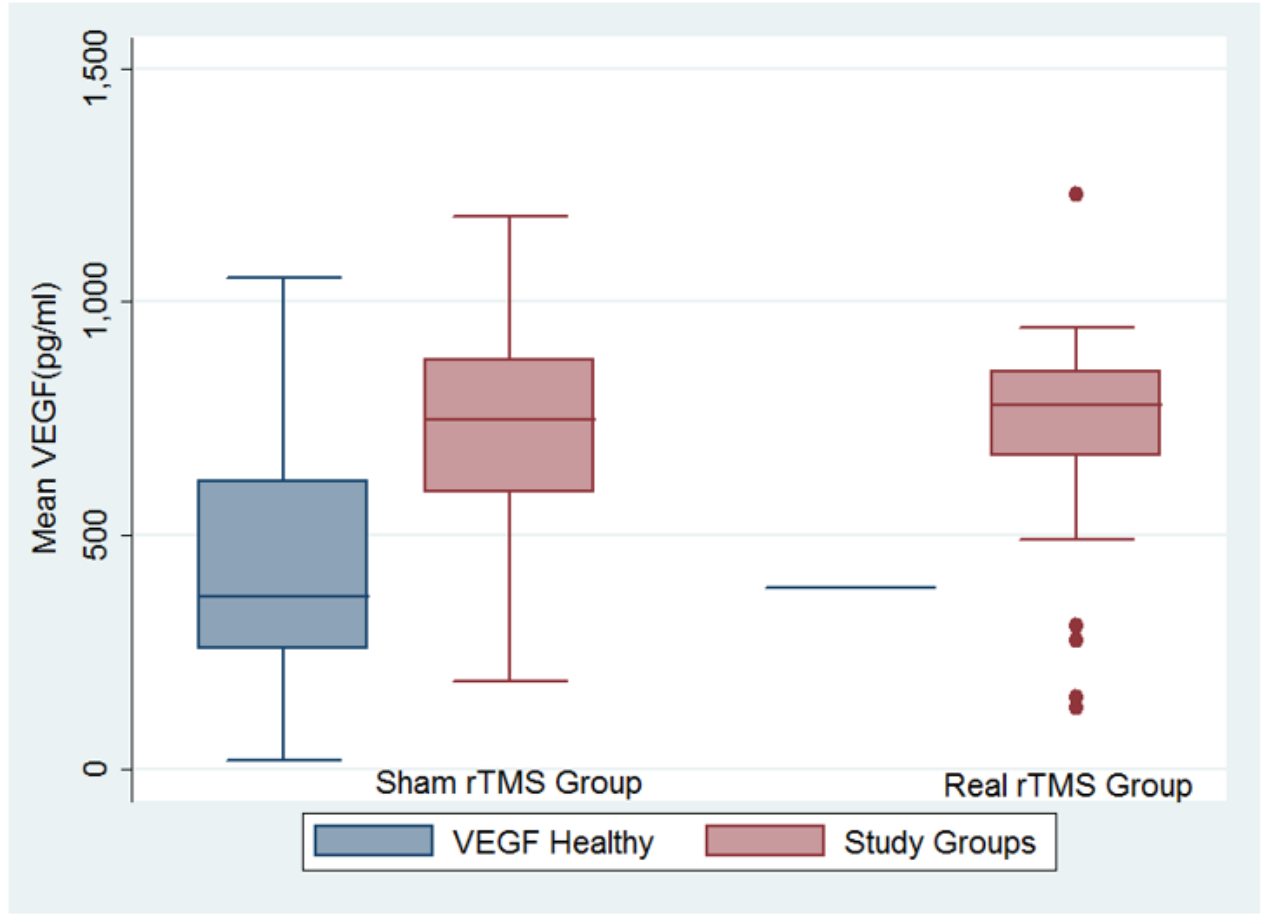

\section{Figure 5}

Whisker plot showing median serum VEGF $(\mathrm{pg} / \mathrm{ml})$ change at recruitment between the Sham rTMS Group, $\mathrm{N}=50$, Real $\mathrm{rTMS}$ Group, $\mathrm{N}=50$ \& Healthy volunteers, $\mathrm{N}=50$

\section{Supplementary Files}

This is a list of supplementary files associated with this preprint. Click to download.

- Table1..docx

- Table2.docx

- SupplementaryBMCrevised.docx

- Table3.docx 